\title{
Werthaltungen, Visionen, Strategien
}

In Workshops und Klausurtagungen hat der Zentralvorstand der FMH seine Werthaltungen, Visionen und Strategien diskutiert, aktualisiert und formuliert - und in einem Papier in eine verdichtete Form gebracht. Dieses enthält die folgenden Grundsatzüberlegungen und Bekenntnisse:

Für Ärztinnen und Ärzte stehen Patientinnen und Patienten im Vordergrund - für die FMH auch, daneben vor allem aber ihre Mitglieder und deren Interessen, also die Ärzteschaft in ihrer ganzen Pluralität. Es geht darum, möglichst gute Bedingungen zu schaffen für die Ausübung des Berufes, es geht - nebst den Dienstleistun- gen, die das Generalsekretariat erbringt - um eine starke Interessenvertretung und Identitätsbildung. Die FMH will mit ihren Mitgliedern einen konstruktiven Dialog führen und Bindeglied sein zwischen ihnen und ihrem (auch internationalen!) Umfeld. In den Bereichen «ärztliche Leistung» und «medizinische Versorgung» will die FMH eine führende Stimme sein - unter dem Motto: «Keine Gesundheitspolitik ohne $\mathrm{FMH!»}$

Das ganze Strategiepapier kann auf unserer Website www.fmh.ch eingesehen werden - in der Rubrik «Über uns» $\rightarrow$ FMH-Zentralvorstand.

\section{Valeurs, visions d'avenir et stratégies}

$\mathrm{Au}$ cours d'ateliers et de séances de réflexion, le Comité central de la FMH a débattu de ses valeurs, visions d'avenir et stratégies, les a réactualisées puis consignées dans un document. Celui-ci contient, sous une forme condensée, les réflexions de base et prises de position suivantes:

Les patients sont au centre des préoccupations des médecins - pour la FMH ce sont ses membres et leurs intérêts, c'est-à-dire le corps médical dans toute sa diversité. Elle a donc pour tâche de créer les meilleures conditions pour l'exercice de la profession. Outre les prestations de service fournies par son secrétariat général, la FMH doit défendre fermement les intérêts du corps médical et promouvoir son identité et son image. Pour ce faire, il lui faut mener un dialogue constructif avec ses membres et faire le lien entre eux et leur environnement (notamment au plan international). Dans les domaines relatifs aux «prestations médicales» et à la «couverture en soins médicaux», la FMH tient à tenir une place de premier rang, selon la devise: «Pas de politique de la santé sans la FMH!»

L'entier document relatif à la stratégie du CC figure sur notre site internet www.fmh.ch, sous la rubrique «Qui sommes-nous?» $\rightarrow$ Le Comité central de la FMH. 